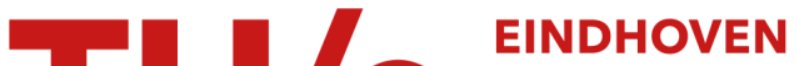 \\ UNIVERSITY OF \\ TECHNOLOGY
}

\section{Near-field speckle imaging of light localization in disordered photonic systems}

\section{Citation for published version (APA):}

Caselli, N., Intonti, F., La China, F., Biccari, F., Riboli, F., Gerardino, A., Li, L., Linfield, E. H., Pagliano, F., Fiore, A., \& Gurioli, M. (2017). Near-field speckle imaging of light localization in disordered photonic systems. Applied Physics Letters, 110(8), 1-6. [081102]. https://doi.org/10.1063/1.4976747

DOI:

10.1063/1.4976747

Document status and date:

Published: 20/02/2017

\section{Document Version:}

Publisher's PDF, also known as Version of Record (includes final page, issue and volume numbers)

\section{Please check the document version of this publication:}

- A submitted manuscript is the version of the article upon submission and before peer-review. There can be important differences between the submitted version and the official published version of record. People interested in the research are advised to contact the author for the final version of the publication, or visit the $\mathrm{DOI}$ to the publisher's website.

- The final author version and the galley proof are versions of the publication after peer review.

- The final published version features the final layout of the paper including the volume, issue and page numbers.

Link to publication

\section{General rights}

Copyright and moral rights for the publications made accessible in the public portal are retained by the authors and/or other copyright owners and it is a condition of accessing publications that users recognise and abide by the legal requirements associated with these rights.

- Users may download and print one copy of any publication from the public portal for the purpose of private study or research.

- You may not further distribute the material or use it for any profit-making activity or commercial gain

- You may freely distribute the URL identifying the publication in the public portal.

If the publication is distributed under the terms of Article 25fa of the Dutch Copyright Act, indicated by the "Taverne" license above, please follow below link for the End User Agreement:

www.tue.nl/taverne

Take down policy

If you believe that this document breaches copyright please contact us at:

openaccess@tue.nl

providing details and we will investigate your claim. 


\section{Near-field speckle imaging of light localization in disordered photonic systems}

Niccolò Caselli, Francesca Intonti, Federico La China, Francesco Biccari, Francesco Riboli, Annamaria Gerardino, Lianhe Li, Edmund H. Linfield, Francesco Pagliano, Andrea Fiore, and Massimo Gurioli

Citation: Appl. Phys. Lett. 110, 081102 (2017); doi: 10.1063/1.4976747

View online: http://dx.doi.org/10.1063/1.4976747

View Table of Contents: http://aip.scitation.org/toc/apl/110/8

Published by the American Institute of Physics

\section{Articles you may be interested in}

Optically controllable nanobreaking of metallic nanowires

Applied Physics Letters 110, 081101 (2017); 10.1063/1.4976947

The effects of magnesium doping on the modal loss in AIGaN-based deep UV lasers

Applied Physics Letters 110, 081103 (2017); 10.1063/1.4977029

Designing an efficient rectifying cut-wire metasurface for electromagnetic energy harvesting Applied Physics Letters 110, 083901 (2017); 10.1063/1.4976804

Enhancing light transmission through a disordered waveguide with inhomogeneous scattering and loss Applied Physics Letters 110, 021103 (2017); 10.1063/1.4973459

Surface emitting thermally assisted polaritonic light-emitting device

Applied Physics Letters 110, 081108 (2017); 10.1063/1.4976585

Electrically driven and electrically tunable quantum light sources Applied Physics Letters 110, 071102 (2017); 10.1063/1.4976197

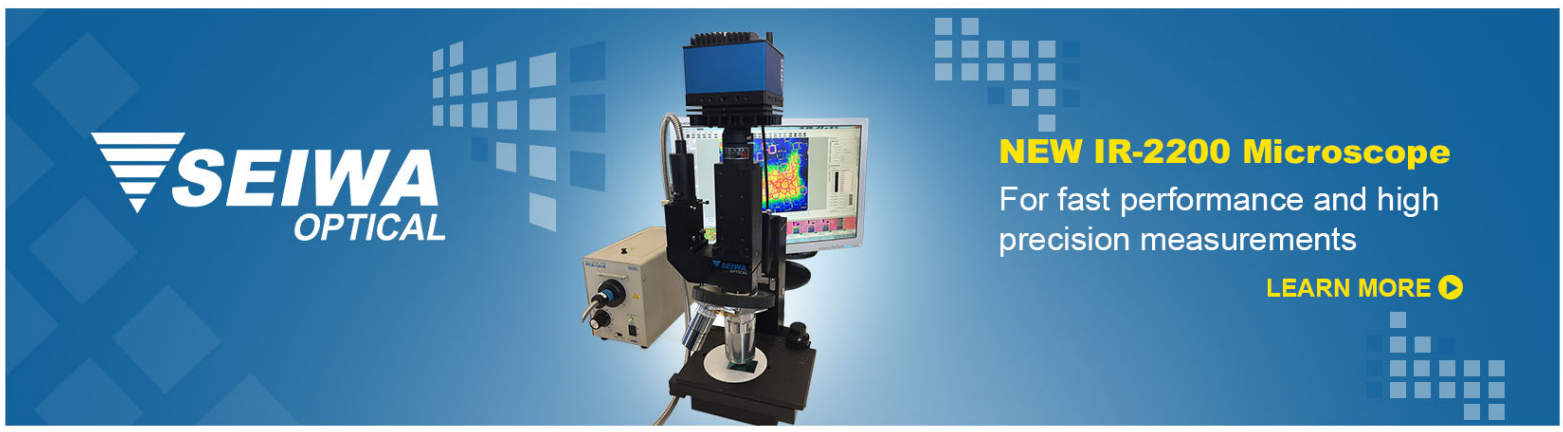




\title{
Near-field speckle imaging of light localization in disordered photonic systems
}

\author{
Niccolò Caselli, ${ }^{1,2, a)}$ Francesca Intonti, ${ }^{1,2}$ Federico La China, ${ }^{1,2}$ Francesco Biccari, ${ }^{1,2}$ \\ Francesco Riboli, ${ }^{3}$ Annamaria Gerardino, ${ }^{4}$ Lianhe Li, ${ }^{5}$ Edmund H. Linfield, ${ }^{5}$ \\ Francesco Pagliano, ${ }^{6}$ Andrea Fiore, ${ }^{6}$ and Massimo Gurioli ${ }^{1,2}$ \\ ${ }^{1}$ European Laboratory for Nonlinear Spectroscopy, via Nello Carrara 1, I-50019 Sesto Fiorentino (FI), Italy \\ ${ }^{2}$ Department of Physics, University of Florence, via Sansone 1, I-50019 Sesto Fiorentino (FI), Italy \\ ${ }^{3}$ Department of Physics, University of Trento, via Sommarive 14, I-38123 Povo (TN), Italy \\ ${ }^{4}$ Institute of Photonics and Nanotechnology, CNR, Via Cineto Romano, 42, I-00156 Roma, Italy \\ ${ }_{5}^{5}$ School of Electronic and Electrical Engineering, University of Leeds, Leeds LS2 9JT, United Kingdom \\ ${ }^{6}$ Department of Applied Physics and Institute for Photonic Integration, Eindhoven University of Technology, \\ $5600 \mathrm{MB}$ Eindhoven, The Netherlands
}

(Received 7 November 2016; accepted 4 February 2017; published online 22 February 2017)

\begin{abstract}
Optical localization in strongly disordered photonic media is an attractive topic for proposing novel cavity-like structures. Light interference can produce random modes confined within small volumes, whose spatial distribution in the near-field is predicted to show hot spots at the nanoscale. However, these near-field speckles have not yet been experimentally investigated due to the lack of a high spatial resolution imaging techniques. Here, we study a system where the disorder is induced by random drilling air holes in a GaAs suspended membrane with internal InAs quantum dots. We perform deep-subwavelength near-field experiments in the telecom window to directly image the spatial distribution of the electric field intensity of disordered-induced localized optical modes. We retrieve the near-field speckle patterns that extend over few micrometers and show several single speckles of the order of $\lambda / 10$ size. The results are compared with the numerical calculations and with the recent findings in the literature of disordered media. Notably, the hot spots of random modes are found in proximity of the air holes of the disordered system. Published by AIP Publishing.
\end{abstract}

[http://dx.doi.org/10.1063/1.4976747]

Dielectric disordered materials are ubiquitous in nature, ranging from marble to clouds and fog up to biological tissues. The description of light behavior inside them has been a long-standing subject in optics. ${ }^{1}$ In the disordered media, light undergoes multiple scattering, which is relevant not only to basic physics but also for technological applications. ${ }^{2}$ The stimulated emission in the multiple light scattering regime has opened the field of random lasers. ${ }^{3-6}$ For photovoltaic applications, a disordered distribution of air holes was proved to improve the absorption of thin-film. ${ }^{7}$ Since light does not lose its coherence while undergoing elastic scattering, it interferes producing speckle patterns, consisting of a complex collection of dark and bright electric field intensity spots. This effect blurs any direct vision through a disordered dielectric medium, but by acquiring the far-field speckle pattern and using adaptive phase masks, it is possible to retrieve images through opaque layers. ${ }^{8}$ Generally, whenever the photon mean free path is small enough, multiple scattering suppresses light propagation, producing localized modes inside the disordered dielectric medium. ${ }^{9,10}$ Localization in disordered media is an interference effect; thus, it can also be observed for electrons, for which indeed the concept of Anderson localization was originally introduced. ${ }^{11,12}$ The localization of light has been discussed in many disordered photonic systems, and it is characterized by modes with a high level of spatial

\footnotetext{
${ }^{a}$ Current address: Instituto de Ciencia de Materiales de Madrid - CSIC, Calle Sor Juana Inés de la Cruz, 3, 28049 Madrid, Spain. Electronic mail: caselli@lens.unifi.it
}

confinement and long lifetimes inside the sample. In three dimensional systems the realization of localized modes is extremely challenging. ${ }^{1,9,10}$ Indeed, many recent reports, which aim at studying, engineering and taking advantage of disordered localized modes, have focused on one or twodimensional systems. ${ }^{13-15}$ Besides the huge impact of Anderson localization on basic physics, the concept of spatially localized modes in a strongly disordered photonic structure has triggered the exploitation of disordered systems to enhance light matter interaction ${ }^{16}$ and to open new quantum electrodynamic scenarios. ${ }^{17}$

All these predictions of light localization share a common feature: within the confinement volume the mode amplitude has hot spots, which are a direct manifestation of interference in the near-field. The confined modes show amplitude modulation from bright to dark, which resemble the well-known speckle patterns in the far-field scattering from any disordered media. ${ }^{18}$ Hereafter, we will exploit this similarity by denominating the hot spots localized at the nanoscale as near-field speckles. These speckles can also be found on the surface of disordered samples that do not localize light. ${ }^{19-21}$ The study of the correlation length of the nearfield speckles allowed for extracting some characteristics of the bulk samples, as the refractive index, ${ }^{19}$ or discriminating between materials with different internal microscopic structures. ${ }^{21}$ So far, the direct observation of near-field speckles of a strongly localized optical state in disordered photonic system has not been reported. In addition, the spatial distribution of near-field speckles localized in air or in dielectric 
regions is fundamental for the application of random modes. Theoretical predictions in the $\mathrm{THz}$ region for random air holes in dielectric membranes stated that random transverseelectric (TE) modes have near-field speckles localized inside the air holes, in contrary to the transverse-magnetic (TM) modes, which are mainly localized in the dielectric media. ${ }^{22}$

In this paper, we address the near field imaging of isolated photonic modes localized in a strongly scattering disordered medium. In particular, we investigate a photonic structure of disordered air pores realized on a high refractive index semiconductor slab, where the randomness in the pore position is solely limited by their finite size. The sample under study is fabricated on a $320 \mathrm{~nm}$ thick GaAs suspended membrane of $25 \mu \mathrm{m} \times 25 \mu \mathrm{m}$ size. Electron beam lithography and reactive ion etching are used to pattern the membrane with the nominally identical air holes (diameter $220 \mathrm{~nm}$ ) giving an overall filling fraction of $30 \%$. Finally, wet etching of the bottom AlGaAs sacrificial layer is used to release the membrane. We designed the position of the holes by a random sequential addition generator, ${ }^{15}$ and we imposed a minimum distance (1.3 hole diameters) between the centers of the nearest neighboring holes in order to avoid merging between adjacent holes during the fabrication process. Therefore, even though the sample is not fully random, hereafter, we will use the denomination random system/random modes both to stress the maximum practical randomness of our realization and to distinguish our sample from disordered media with higher spatial correlation. ${ }^{23}$ This system has previously demonstrated to support localized modes in the telecom wavelength range, which is relevant for photonic applications. ${ }^{15,24}$ We use the emission of embedded quantum dots (QDs) to pump the randomly generated modes. In fact, three layers of high-density $\left(300 \mu \mathrm{m}^{-2}\right)$ InAs QDs were grown in the middle of the membrane by molecular beam epitaxy.

In order to check the spatial distribution of the random modes, we employ a near-field illumination that is crucial to map strongly localized light. Then, we exploit the local perturbation induced by the dielectric near-field probe, which results in a spectral tuning of the photonic modes, to retrieve the distributions of random modes with a deep-subwavelength spatial resolution..$^{25,26}$ The experimental apparatus is a commercial near-field optical microscope (SNOM), TwinSNOM, Omicron NanoTechnology GmbH, Germany. The QDs are excited through a dielectric near-field tip with a $780 \mathrm{~nm}$ diode laser at a power equal to $60 \mu \mathrm{W}$. The photoluminescence (PL) signal is collected through the same tip, dispersed by a spectrometer and detected by an InGaAs array. The high density of QDs guarantees a spatially homogeneous emission in a broad spectral range, from $1.10 \mu \mathrm{m}$ to $1.35 \mu \mathrm{m}$, all over the sample. Then, the observation of nearly monochromatic bright spots by measuring the map of the PL can be directly related to the presence of a localized mode, which increases the QD radiative rate. By measuring the PL intensity at each tip position, we obtain a hyperspectral imaging of the photonic random modes with a combined spatial and spectral resolution of $250 \mathrm{~nm}$ and $0.1 \mathrm{~nm}$ for the optical signal, respectively. In addition, the SNOM experiments also provide morphological information on the sample surface (with a spatial resolution better than $50 \mathrm{~nm}$ ) via the mechanical feedback signal of the shear-force probe, which allows a precise alignment between the optical signal and the positions of the scatterers. In order to achieve a high fidelity and direct mapping of the electric field intensity of the random modes and at the same time to increase the spatial resolution of the measurement, we exploit the perturbation imaging technique. ${ }^{25}$ The experimental optical maps demonstrate that individual random modes exhibit the near-field speckles with the size of about $100 \mathrm{~nm}$. The results are compared to the 3D finite difference time domain (FDTD) simulations, leading to a nice agreement and demonstrating the speckles concentration around the scattering centers. In the simulations, the system is excited by pulsed point like dipoles (in the spectral range 1.1-1.4 $\mu \mathrm{m}$ ) with in plane polarization placed in the middle of the GaAs membrane in order to reproduce the real system, which is excited by the PL signal of InAs quantum dots self-assembled in the middle of the suspended membrane.

In Fig. 1(a), we report the SEM image of the sample along with the schematics of the mode distribution and of the near-field probe on top of a random localized mode. The tip raster scans the sample surface and collects the PL point by
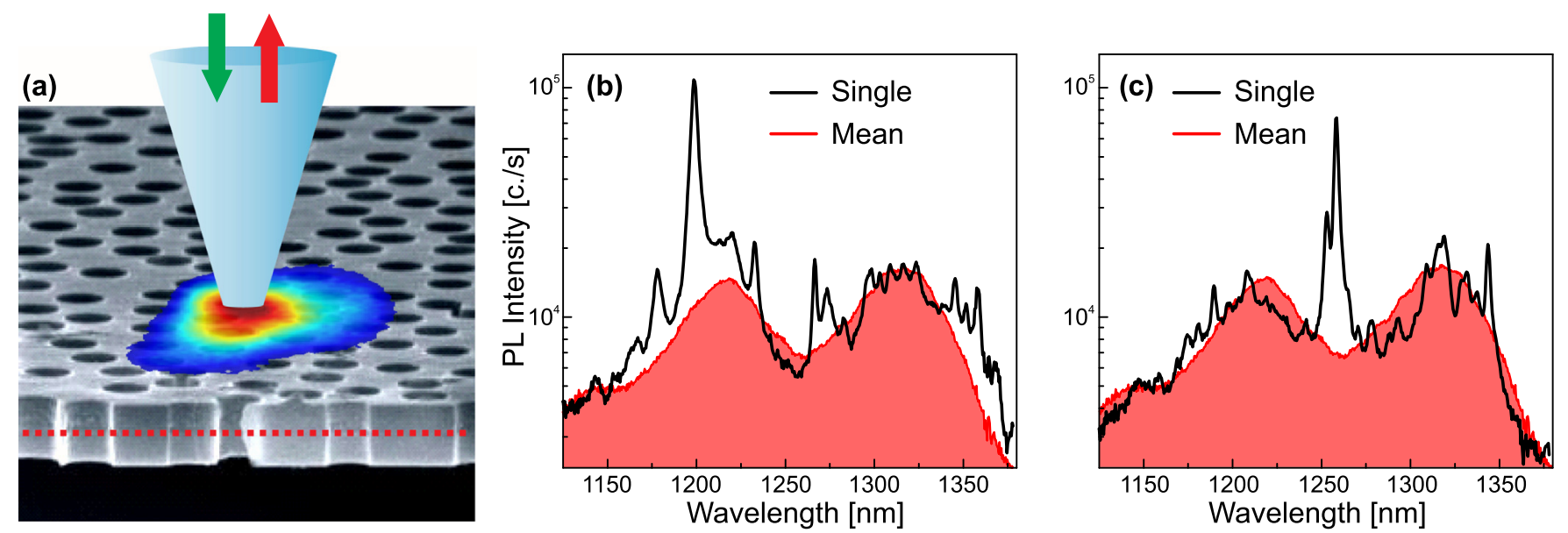

FIG. 1. (a) Schematics of the experimental setup in illumination/collection through the near-field probe. SEM image of the GaAs suspended membrane with disordered scatterers, where QDs are represented by red dots in the middle of the membrane. The near-field probe interacts with a random localized mode inside the disordered media (represented in a blue to red color scale) and collects the light that leaks out. (b) and (c) Near-field spectra reported in semi-log scale collected in two different positions of the sample surface (black lines). The peaks corresponding to the optical random modes are superimposed to the broadband emission of QDs (red area) evaluated as the mean spectrum acquired in the whole SNOM scan. 
point. Typical spectra are reported in a semi-log scale in Figs. 1(b) and 1(c) (black lines) for two generic positions and compared to the mean spectra of the entire scan (red curves), which represent the PL of the internal QDs. The two broad bands are centered around $1320 \mathrm{~nm}$ and $1220 \mathrm{~nm}$, corresponding to the transitions towards the ground state and the first excited state of the InAs QDs, respectively. In the single spectra, sharp resonances, related to the electromagnetic modes of the system, are clearly observed. The modes corresponding to these sharp resonances extend over few micrometers in the slab plane, covering few scattering elements, and they are spectrally sparse over the full emission range of the QDs, with the quality factor ranging from 200 to 400 . The estimated density is around 3 modes per $\mu \mathrm{m}^{2}$, denoting that the investigated sample is inducing many disordered localized modes. So far, due to the large spectral range under investigation, we find that the resonances can be spectrally isolated, assuring that these modes fulfill the Thouless criterion for the localized regime. ${ }^{2}$ Other statistical analysis, such as the probability density function, gives the same conclusion. ${ }^{15}$ Let us stress that our near-field approach directly shows that the observed resonances are spatially confined, which is an evident and unambiguous proof that, in the investigated wavelength range, the sample supports localized photonic modes. Therefore, within the near-field approach, a statistical analysis is not mandatory for concluding if the sample is in the confined or in the diffusive regime. This agrees with the claim reported in Ref. 18, based on the similarity of the far-field speckle patterns obtained with different excitation configurations that it is possible to discriminate between a localized and a diffusive sample, just by considering a single realization of disorder without performing further statistical analysis.

For each confined mode, we reconstruct the PL map by evaluating the signal collected by the SNOM apparatus at a fixed wavelength as a function of the position. Generally, the maps show several spots that arise from different and spectrally overlapping localized modes. A map of an intense and spectrally isolated mode is reported in Fig. 2(a). Although analyzing the PL maps may lead to a statistical analysis, since it concerns many random modes, it is quite complicated to use them for addressing the near-field speckle of an individual mode. In order to achieve this goal, we perform a mode analysis by fitting each PL spectral peak with the Lorentzian lineshape. The peak amplitude provides a PL intensity map, as reported in Fig. 2(b), where the map is superimposed to the sample morphology. The mode is localized in a few microns, but the $250 \mathrm{~nm}$ resolution of our dielectric tip does not allow us to isolate the near-field speckles, leading to a measure of the mode envelope only. In order to overcome this limitation, we use the dielectric tip perturbation as a tool to image the mode electric field intensity distribution. In fact, it is known that the presence of a dielectric perturbation on the photonic resonator modifies the resonant wavelength of the confined mode, producing a red shift. ${ }^{25-27}$ This spectral shift has been proved to be more pronounced if the perturbation acts in the positions where the localized mode has a maximum electric field intensity, i.e., the tip induced spectral shift is directly proportional to the mode electric field intensity. Therefore, this effect has been largely used in photonic cavities either to exploit them as a sensor or to use the shift as a direct measure of the local electric field intensity. ${ }^{27-29}$ In Fig. 2(c), we show near-field spectra acquired on three different points, labelled as A, B and C in Fig. 2(b).

Indeed, besides a variation in intensity, they show a sizable spectral shift, that, between the positions B and C, is $\Delta \lambda=0.50 \mathrm{~nm}$. Since the induced maximum shift is inversely proportional to the mode volume, a meaningful shift is also a confirmation of the small modal volume of localized modes in the random systems on slab. In addition, since the proportionality between the mode shift and the mode electric field intensity at the position of the nanometric perturbation (the SNOM tip) is an exact result, the perturbation imaging is a more sensitive and high fidelity tool to retrieve the mode electric field intensity. Moreover, the resolution the perturbation imaging is a more sensitive and high fidelity tool to retrieve the mode electric field intensity, with respect to the PL imaging. ${ }^{25,28}$ All these considerations lead us to exploit the spectral shift for a direct mapping of individual localized random modes. Figure 3(a) shows a three dimensional plot of the spatial distribution of the experimental electric field intensity of a mode centered at $1338 \mathrm{~nm}$. The map is obtained by reporting, at every tip location, the spectral position of the mode. Inside the envelope of the mode, we observe a complex pattern of near-field speckles with typical dimension around $100 \mathrm{~nm}$. In Fig. 3(b), the FDTD calculation of the electric field intensity of a typical random mode is reported. The calculation is performed on a disordered medium that, nominally, corresponds to the design of the investigated sample. The reported intensity profile is
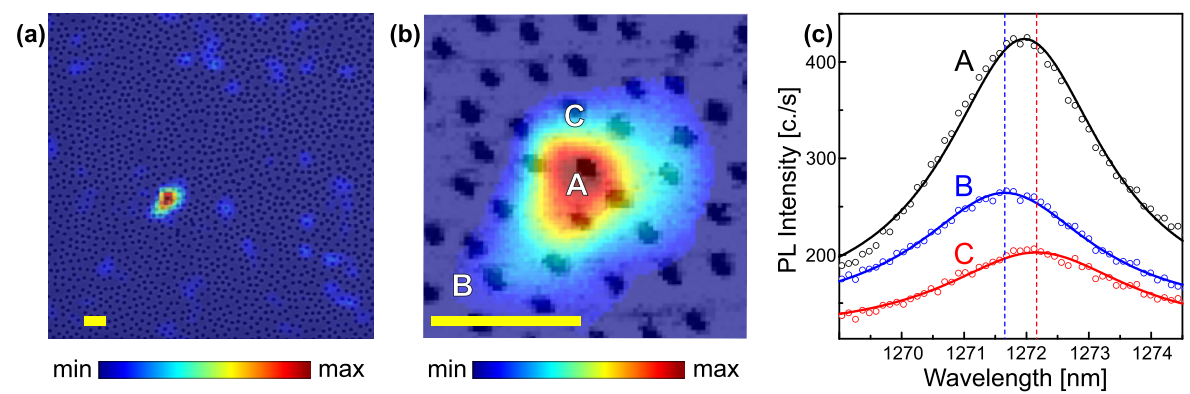

FIG. 2. (a) PL near-field intensity map of the sample evaluated at wavelength of $1272 \mathrm{~nm}$, where a clear peak emerges in some of the acquired spectra. The optical map is superimposed to the SEM image of the sample. (b) PL amplitude map for the mode evaluated in (a), obtained by fitting the spectra with a Lorentzian lineshape. It is superimposed to the topography reconstructed by the SNOM microscope. The scale bar in both (a) and (b) is $1 \mu \mathrm{m}$. (c) Near-field spectra (scattered circles) collected in the positions A, B and C labelled in (b). The lines are the Lorentzian fits of the data. The vertical dashed lines highlight the peak wavelength of the spectra $\mathrm{B}$ and $\mathrm{C}$, denoting a spectral shift $\Delta \lambda=0.50 \mathrm{~nm}$. 

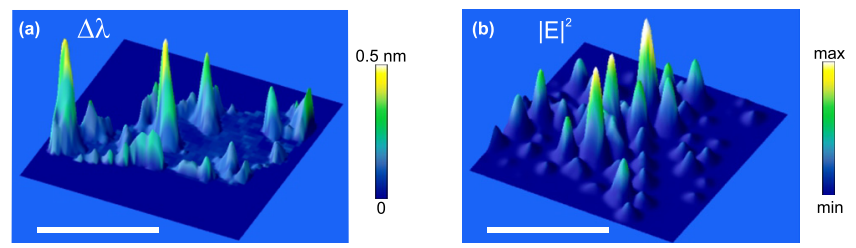

FIG. 3. (a) Three-dimensional map of the spectral shift of a peak whose wavelength is around $1338 \mathrm{~nm}$, showing the near-field speckle patterns of a localized random mode. (b) Three-dimensional distribution of the electric field intensity of a mode evaluated by the FDTD calculation in a medium with the same design characteristics of the experimentally investigated sample. The scale bar is $1 \mu \mathrm{m}$.

obtained after calculation time long enough that, in the wavelength range of interest, there is only one mode remaining. Although a quantitative comparison between the mode distribution of Figs. 3(a) and 3(b) is outside the possibility of this analysis, due to the unintentional disorder introduced during the nanofabrication, in both speckle distributions we find strong similarities regarding the random ensemble of hot spots that exhibit the same overall extension and the same single speckle dimension.

Intuitively, the electric field of random modes, depending on their quasi-TE or quasi-TM nature and on the nature of the scatterers (air holes in a dielectric slab or dielectric pillars in air), should result prevalently confined either in the dielectric regions or in the air ones. ${ }^{6,22,30}$ Notably, for circular air holes etched in high refractive-index materials, the quasi-TE modes appear more efficiently confined in the air regions. $^{22}$ In order to get a quantitative comparison between the experiment and the theory, we study where the quasi-TE random modes are more efficiently confined. We estimated the average intensity $\left(\mathrm{I}_{\mathrm{N}}\right)$ of the electric field $(\mathrm{E})$ in different regions by $\mathrm{I}_{\mathrm{N}}=\frac{1}{\mathrm{~S}_{\mathrm{N}}} \frac{\int_{\mathrm{S}_{\mathrm{i}}} \mathrm{E}^{2} \mathrm{dV}}{\int_{\mathrm{Tot}} \mathrm{E}^{2} \mathrm{dV}}$, where $\mathrm{i}=$ Air or $\mathrm{i}=$ Die for air and dielectric regions, respectively. We quantify the mode concentration by $\mathrm{R}=\frac{\mathrm{I}_{\text {Air }}}{\mathrm{I}_{\text {Die }}}$. Through the FDTD simulations in the regions of $3 \times 3 \mu \mathrm{m}^{2}$ size, we investigated 11 localized random modes with emission ranging from $1.15 \mu \mathrm{m}$ to $1.40 \mu \mathrm{m}$; two of them are reported in Figure 4. As expected, ${ }^{22}$ these modes are mainly trapped in the air-holes, where they exhibit $\mathrm{R}=(2.3 \pm 0.2)$, which corresponds to an average electric field intensity in the air-region more than twice larger than the one found in the dielectric-region. On the other hand, the near-field PL experiments allow us to correlate optical near-field maps with morphological information recorded during the hyperspectral scanning. We draw the attention on two random modes whose wavelength is about $1272 \mathrm{~nm}$ and $1338 \mathrm{~nm}$. The experimental twodimensional spectral-shift maps of the two random modes are given in Figs. 4(a) and 4(b), respectively, evaluated by the Lorentzian lineshape fitting. These maps are superimposed to the topography maps. They show near-field speckle patterns exhibiting hot spots with full width at half maximum of the order of $100 \mathrm{~nm}$ and tightly localized in close proximity of the air pores. They exhibit $\mathrm{R}=(1.9 \pm 0.3)$ in agreement with the value obtained by FDTD. In Figs. 4(c) and 4(d), we report the electric field intensity distributions calculated by FDTD for two modes whose peak wavelengths are in the range of the experimental ones, using the nominal design hole pattern. The maps are superimposed to the design of the sample. They show near-field-speckles in close proximity of the air pore scattering centres. This feature is shown in more detail in Figs. 4(e)-4(f), where we report the line profile cuts of the experimental map of Fig. 4(b) and of the theoretical map of Fig. 4(d), respectively. The optical speckles are found in correspondence with the hole positions. The FDTD calculated speckles are also inside the photonic pores, particularly in close proximity to the internal hole surfaces. Therefore, the outcomes of the SNOM experiments related to the size and localization of the near-field speckles of the random modes accurately agree with the theoretical predictions.

It could be questioned whether the localization in the air pores represents a limitation for the exploitation of random modes in optoelectronic devices, due to the reduction of the overlap between the electric field and the emitters/absorbers. Naturally, the localization in air is a limitation for devices based on emitters or absorbers integrated inside the slab. For example, if the goal is increasing the absorption of silicon
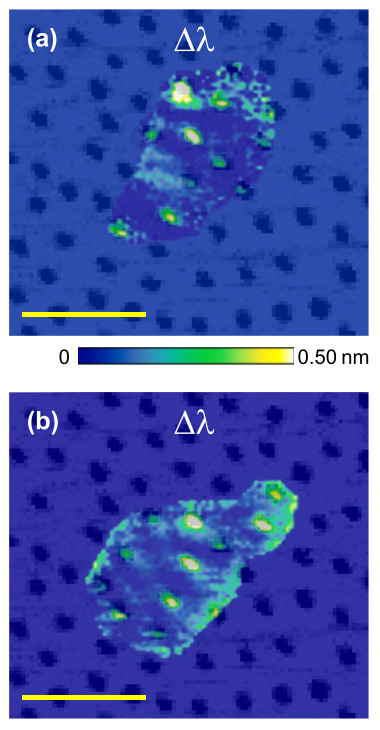

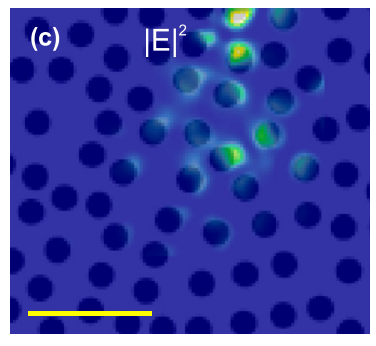

$\min$

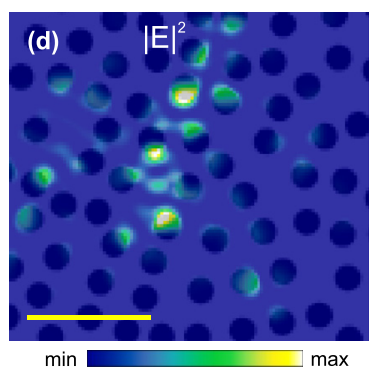

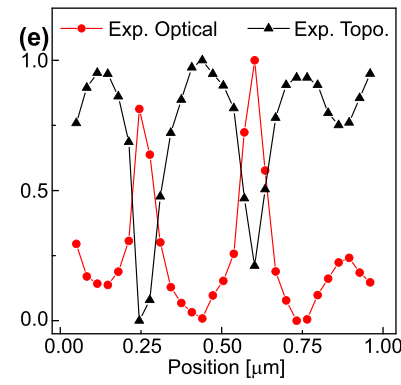

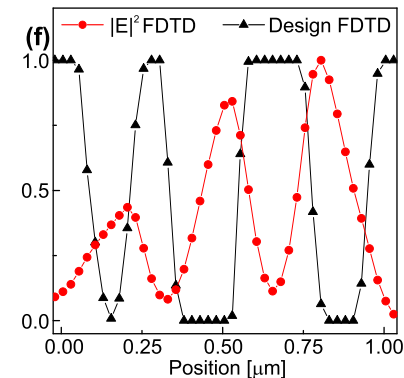

FIG. 4. (a) and (b) Spectral shift $(\Delta \lambda)$ maps obtained by evaluating the peak position by the Lorentzian fits, for the modes centered around $1272 \mathrm{~nm}$ and $1338 \mathrm{~nm}$, respectively. (c) and (d) Electric field intensity maps obtained by the FDTD calculations, for the modes centered around $1272 \mathrm{~nm}$ and $1338 \mathrm{~nm}$, respectively. The scale bar is $1 \mu \mathrm{m}$. (e) Normalized line profiles of the experimental spectral shift map (red dots) and of the experimental topography map (black triangles) reported in (b). (f) Normalized line profiles of the FDTD calculated electric field intensity map (red dots) and of the dielectric design employed in the calculation (black triangles) reported in (d). 
thin-film solar cells, a geometry that confines light mainly in the dielectric regions is preferable. ${ }^{7}$ At the same time, the near-field hot spots localized in air are required for hybrid solution in the $\mathrm{Si}$ photonics, where the emitters/absorbers such as carbon nanotubes need to be integrated on top of the photonic structure or in the air region. ${ }^{31}$ Similarly, in view of photonic sensors, a localization of random modes in the air region is required for increasing the sensitivity. ${ }^{29}$ Our results point out for a detailed theoretical study (out of the scope of this paper) concerning the role of the disordered design in localizing the electric field in air or dielectric regions.

In conclusion, we imaged the near-field speckle pattern of photonic modes strongly localized in the disordered medium suspended on membrane. We exploited the quantum dot photoluminescence for performing perturbation imaging with deep-subwavelength spatial resolution, which allows for a direct mapping of the electric field intensity of the random modes. They extend over 1-2 $\mu \mathrm{m}$, exhibiting single near-field speckles of $100 \mathrm{~nm}$ size, at wavelengths around $1300 \mathrm{~nm}$. The hot spot speckles, in agreement with the theoretical predictions, are strongly localized in the inner edges of the scattering centers for all the investigated random modes. Our findings demonstrate that disordered systems can build up localized photonic modes exhibiting strong spatial non-uniformity with very small spatial features. This lay the grounds for the exploitation of random localization in optoelectronic devices like more efficient silicon thin-film solar cells, ${ }^{7}$ random lasers ${ }^{32}$ and sensors. ${ }^{33}$

This work was supported by the FET project FP7 618025 CARTOON. This work is part of the research programme of the Foundation for Fundamental Research on Matter (FOM), which is financially supported by the Netherlands Organisation for Scientific Research (NWO), and it is also supported by the Dutch Technology Foundation STW, applied science division of NWO, the Technology Program of the Ministry of Economic Affairs under Project No. 10380. F.B. acknowledges funding from the Italian Ministry for Education, University and Research within the Futuro in Ricerca (FIRB) program (project DeLIGHTeD, Protocollo RBFR12RS1W).

${ }^{1} \mathrm{P}$. Sheng, Introduction to Wave Scattering, Localization and Mesoscopic Phenomena (Springer, 2006).

${ }^{2}$ D. S. Wiersma, "Disordered photonics," Nat. Photonics 7, 188-196 (2013).

${ }^{3}$ H. Cao, Y. G. Zhao, H. C. Ong, S. T. Ho, J. Y. Dai, J. Y. Wu, and R. P. H. Chang, "Ultraviolet lasing in resonators formed by scattering in semiconductor polycrystalline films," Appl. Phys. Lett. 73, 3656-3658 (1998).

${ }^{4}$ H. Cao, Y. G. Zhao, S. T. Ho, E. W. Seelig, Q. H. Wang, and R. P. H. Chang, "Random laser action in semiconductor powder," Phys. Rev. Lett. 82, 2278 (1999).

${ }^{5}$ D. S. Wiersma, "The physics and applications of random lasers," Nat. Phys. 4, 359-367 (2008).

${ }^{6} \mathrm{P}$. Sebbah and C. Vanneste, "Random laser in the localized regime," Phys. Rev. B 66, 144202 (2002).

${ }^{7}$ K. Vynck, M. Burresi, F. Riboli, and D. S. Wiersma, "Photon management in two-dimensional disordered media," Nat. Mater. 11, 1017-1022 (2012).

${ }^{8}$ J. Bertolotti, E. G. van Putten, C. Blum, A. Lagendijk, W. L. Vos, and A. P. Mosk, "Non-invasive imaging through opaque scattering layers," Nature 491, 232-234 (2012).

${ }^{9}$ D. S. Wiersma, P. Bartolini, A. Lagendijk, and R. Righini, "Localization of light in a disordered medium," Nature 390, 671-673 (1997).
${ }^{10}$ T. Sperling, W. Bührer, C. M. Aegerter, and G. Maret, "Direct determination of the transition to localization of light in three dimensions," Nat. Photonics 7, 48-52 (2012).

${ }^{11}$ A. F. Ioffe and A. R. Regel, "Non-crystalline, amorphous and liquid electronic semiconductors," Prog. Semicond. 4, 237 (1960).

${ }^{12} \mathrm{P}$. W. Anderson, "Absence of diffusion in certain random lattices," Phys. Rev. 109, 1492-1505 (1958).

${ }^{13}$ S. Karbasi, C. R. Mirr, P. G. Yarandi, R. J. Frazier, K. W. Koch, and A. Mafi, "Observation of transverse Anderson localization in an optical fiber," Opt. Lett. 37, 2304-2306 (2012).

${ }^{14}$ T. Schwartz, G. Bartal, S. Fishman, and M. Segev, "Transport and Anderson localization in disordered two-dimensional photonic lattices," Nature 446(7131), 52-55 (2007).

${ }^{15}$ F. Riboli, N. Caselli, S. Vignolini, F. Intonti, K. Vynck, P. Barthelemy, A. Gerardino, L. Balet, L. H. Li, A. Fiore, M. Gurioli, and D. S. Wiersma, "Engineering of light confinement in strongly scattering disordered media," Nat. Mater. 13(7), 720-725 (2014).

${ }^{16}$ R. Sapienza, P. Bondareff, R. Pierrat, B. Habert, R. Carminati, and N. F. van Hulst, "Long-tail statistics of the purcell factor in disordered media driven by near-field interactions," Phys. Rev. Lett. 106, 163902 (2011).

${ }^{17}$ L. Sapienza, H. Thyrrestrup, S. Stobbe, P. D. Garcia, S. Smolka, and P. Lodahl, "Cavity quantum electrodynamics with Anderson-localized modes," Science 327, 1352 (2010).

${ }^{18}$ O. Leseur, R. Pierrat, J. J. Sáenz, and R. Carminati, "Probing twodimensional Anderson localization without statistics," Phys. Rev. A 90, 053827 (2014).

${ }^{19}$ V. Emiliani, F. Intonti, M. Cazayous, D. S. Wiersma, M. Colocci, F. Aliev, and A. Lagendijk, "Near-field short range correlation in optical waves transmitted through random media," Phys. Rev. Lett. 90, 250801 (2003).

${ }^{20}$ A. Apostol and A. Dogariu, "Spatial correlations in the near-field of random media," Phys. Rev. Lett. 91, 093901 (2003).

${ }^{21}$ V. Parigi, E. Perros, G. Binard, C. Bourdillon, A. Matre, and R. Carminati, "Near-field to far-field characterization of speckle patterns generated by disordered nanomaterials," Opt. Express 24, 7019 (2016).

${ }^{22}$ H. K. Liang, B. Meng, G. Liang, J. Tao, Y. Chong, Q. J. Wang, and Y. Zhang, "Electrically pumped mid-infrared random lasers," Adv. Mater. 25, 6859-6863 (2013).

${ }^{23}$ G. M. Conley, M. Burresi, F. Pratesi, K. Vynck, and D. S. Wiersma, "Light transport and localization in two-dimensional correlated disorder," Phys. Rev. Lett. 112, 143901 (2014).

${ }^{24}$ N. Caselli, F. Riboli, F. Intonti, F. La China, F. Biccari, A. Gerardino, and M. Gurioli, "Spatial steadiness of individual disorder modes upon controlled spectral tuning," APL Photonics 1, 041301 (2016).

${ }^{25}$ F. Intonti, S. Vignolini, F. Riboli, A. Vinattieri, D. S. Wiersma, M. Colocci, L. Balet, C. Monat, C. Zinoni, L. H. Li et al., "Spectral tuning and near-field imaging of photonic crystal microcavities," Phys. Rev. B 78, 041401(R) (2008).

${ }^{26}$ A. F. Koenderink, M. Kafesaki, B. C. Buchler, and V. Sandoghdar, "Controlling the resonance of a photonic crystal microcavity by a nearfield probe," Phys. Rev. Lett. 95, 153904 (2005).

${ }^{27}$ S. Mujumdar, A. F. Koenderink, T. Sünner, B. C. Buchler, M. Kamp, A. Forchel, and V. Sandoghdar, "Near-field imaging and frequency tuning of a high-Q photonic crystal membrane microcavity," Opt. Express 15(25), 17214-17220 (2007).

${ }^{28}$ N. Caselli, F. Intonti, F. La China, F. Riboli, A. Gerardino, W. Bao, A. Weber-Bargioni, L. Li, E. H. Linfield, F. Pagliano, A. Fiore, and M. Gurioli, "Ultra-subwavelength phase-sensitive Fano-imaging of localized photonic modes," Light: Sci. Appl. 4, e326 (2015).

${ }^{29}$ J. Jágerská, H. Zhang, Z. Diao, N. Le Thomas, and R. Houdré, "Refractive index sensing with an air-slot photonic crystal nanocavity," Opt. Lett. 35, 2523 (2010).

${ }^{30} \mathrm{C}$. Vanneste and P. Sebbah, "Selective excitation of localized modes in active random media," Phys. Rev. Lett. 87, 183903 (2001).

${ }^{31}$ F. Biccari, F. Sarti, N. Caselli, A. Vinattieri, E. Durán-Valdeiglesias, W. Zhang, C. Alonso-Ramos, T. H. C. Hoang, S. Serna, X. Le Roux, E. Cassan, L. Vivien, and M. Gurioli, "Single walled carbon nanotubes emission coupled with a silicon slot-ring resonator," J. Lumin. (available online 2016).

${ }^{32}$ S. Schönhuber, M. Brandstetter, T. Hisch, C. Deutsch, M. Krall, H. Detz, A. M. Andrews, G. Strasser, S. Rotter, and K. Unterrainer, "Random lasers for broadband directional emission," Optica 3, 1035 (2016).

${ }^{33}$ E. Ignesti, F. Tommasi, L. Fini, F. Martelli, N. Azzali, and S. Cavalieri, "A new class of optical sensors: a random laser based device," Sci. Rep. 6, 35225 (2016). 